PROCEEDINGS OF THE

AMERICAN MATHEMATICAL SOCIETY

Volume 139, Number 10, October 2011, Pages 3573-3581

S 0002-9939(2011)10798-6

Article electronically published on February 14, 2011

\title{
THE BALL EMBEDDING PROPERTY OF THE OPEN UNIT DISC
}

\author{
STEFAN BORELL
}

(Communicated by Franc Forstneric)

\begin{abstract}
We prove that the open unit disc $\triangle$ in $\mathbb{C}$ satisfies the ball embedding property in $\mathbb{C}^{2}$; i.e., given any discrete set of discs in $\mathbb{C}^{2}$ there exists a proper holomorphic embedding $\triangle \hookrightarrow \mathbb{C}^{2}$ which passes arbitrarily close to the discs. It is already known that $\mathbb{C}$ does not satisfy the ball embedding property in $\mathbb{C}^{2}$ and that $\triangle$ satisfies the ball embedding property in $\mathbb{C}^{n}$ for $n>2$.
\end{abstract}

\section{INTRODUCTION}

Let $k$ and $n$ be integers such that $0<k<n$. A $k$-ball in $\mathbb{C}^{n}$ is a set $D \subset \mathbb{C}^{n}$ with $D:=V \cap B_{p}$, where $V$ is a $k$-dimensional affine subspace and $B_{p}$ is an open ball centred at a point $p \in V$. A discrete set $D$ of $k$-balls in $\mathbb{C}^{n}$ is a set which is a countable union of $k$-balls in $\mathbb{C}^{n}$ (with pairwise disjoint closures), $D=\bigcup_{j} D_{j}$, with the additional property that each set of the type $\left\{p_{j} \in D_{j} ; j \in \mathbb{N}\right\}$ is a closed and discrete subset of $\mathbb{C}^{n}$.

Let $\varepsilon>0$ and let $D$ be a $k$-ball in $\mathbb{C}^{n}, 0<k<n$. By translation, we may assume that the centre of the $k$-ball is the origin of $\mathbb{C}^{n}$. Let $V$ be the $k$-dimensional linear subspace which contains $D$, and let $V^{\perp}$ be its orthogonal complement. A set $D^{\prime}$ is an $\varepsilon$-perturbation of the $k$-ball $D$ if it is the graph in $\mathbb{C}^{n}$ of a holomorphic map $F: D \rightarrow V^{\perp}$ with $\|F\|<\varepsilon$, i.e.,

$$
D^{\prime}=\left\{(z, F(z)) \in V \oplus V^{\perp} ; z \in D\right\} \subset \mathbb{C}^{n} .
$$

Given a sequence $\varepsilon_{j}$ of positive integers, denoted $\varepsilon=\left(\varepsilon_{1}, \varepsilon_{2}, \varepsilon_{3}, \ldots\right) \in \mathbb{R}_{+}^{\infty}$, and a discrete set $D=\bigcup_{j} D_{j}$ of $k$-balls in $\mathbb{C}^{n}$, an $\varepsilon$-perturbation $D^{\prime}$ of $D$ is a set $D^{\prime}=\bigcup_{j} D_{j}^{\prime}$ such that the closures $\bar{D}_{j}^{\prime}$ are pairwise disjoint and each set $D_{j}^{\prime}$ is an $\varepsilon_{j}$-perturbation of $D_{j}$.

Definition 1.1. A complex space $X$ satisfies the ball embedding property in $\mathbb{C}^{n}$, $0<\operatorname{dim} X<n$, if for any discrete set $D$ of $(\operatorname{dim} X)$-balls in $\mathbb{C}^{n}$ and any $\varepsilon \in \mathbb{R}_{+}^{\infty}$ there exists a proper holomorphic embedding $F: X \hookrightarrow \mathbb{C}^{n}$ such that $F(X)$ contains an $\varepsilon$-perturbation of $D$.

Received by the editors April 27, 2010 and, in revised form, August 26, 2010

2010 Mathematics Subject Classification. Primary 32H02, 32Q40, 32Q45.

The author wishes to thank Frank Kutzschebauch and Erlend Fornæss Wold for fruitful discussions regarding this topic and the reviewer for helpful comments and remarks.

This work was supported by the Swiss National Science Foundation, grants 200020-124668/1 and PBBE2-121066. 
In this paper we prove the following:

Theorem 1.2. The open unit disc in $\mathbb{C}$ satisfies the ball embedding property in $\mathbb{C}^{2}$.

\section{BACKGROUND}

The study of proper holomorphic embedding into complex Euclidean spaces was greatly improved by the ideas and results of Rosay and Rudin. In 10, Rosay and Rudin constructed non-tame discrete sets in $\mathbb{C}^{n}$-a discrete set $E$ in $\mathbb{C}^{n}$ is nontame if no holomorphic automorphism of $\mathbb{C}^{n}$ maps $E$ into a proper linear subspace of $\mathbb{C}^{n}$. Non-tame discrete sets were used by Rosay and Rudin in [11] to construct a non-straightenable proper holomorphic embedding of $\mathbb{C}$ into $\mathbb{C}^{n}, n>2$. This was achieved by constructing a proper holomorphic embedding $F: \mathbb{C} \hookrightarrow \mathbb{C}^{n}$ such that its image contains a non-tame discrete subset of $\mathbb{C}^{n}$. Indeed, such an embedding $F$ is non-straightenable in the sense that there exists no holomorphic automorphism $\varphi$ of $\mathbb{C}^{n}$ for which $\varphi \circ F(\mathbb{C})=\mathbb{C} \times\{0\} \subset \mathbb{C}^{n}$. This result was extended to the case $n=2$ by Forstnerič, Globevnik and Rosay [7] and shows that the well-known result by Abhyankar-Moh [1] and Suzuki [12] - stating that any polynomial embedding of $\mathbb{C}$ into $\mathbb{C}^{2}$ can be made into the canonical inclusion by composition with a polynomial automorphism of $\mathbb{C}^{2}$ - does not hold true in the holomorphic setting.

The ideas and methods of Rosay and Rudin were generalised by Buzzard and Fornæss, who constructed a proper holomorphic embedding $F: \mathbb{C} \hookrightarrow \mathbb{C}^{2}$ such that $\mathbb{C}^{2} \backslash F(\mathbb{C})$ is Kobayashi hyperbolic [4]. This was achieved by constructing a discrete set of discs (1-balls) in $\mathbb{C}^{2}$ and a proper holomorphic embedding such that its image in $\mathbb{C}^{2}$ contains small enough perturbations of the discs. The discs can be chosen in such a way that the complement of any small enough perturbations of the discs is Kobayashi hyperbolic, thus inducing hyperbolicity of $\mathbb{C}^{2} \backslash F(\mathbb{C})$. In turn, the same construction was generalised by Forstnerič [6] to e.g. find proper holomorphic embeddings $F: \mathbb{C}^{k} \hookrightarrow \mathbb{C}^{n}$ for which $\mathbb{C}^{n} \backslash F\left(\mathbb{C}^{k}\right)$ contains no non-degenerate, holomorphic image of $\mathbb{C}^{n-k}$ (with Kobayashi hyperbolicity in the case $k=n-1$ ). In 2, the author and Kutzschebauch made small modifications to the construction of Forstnerič in order to prove that the complement $\mathbb{C}^{n} \backslash F\left(\mathbb{C}^{k}\right)$ can be made $(n-k)$-Eisenman hyperbolic (Eisenman hyperbolicity was introduced in [5]).

The results mentioned in the previous paragraph are proven by inductively choosing $k$-balls in $\mathbb{C}^{n}$ and modifying embeddings in such a way that the end results are embeddings whose images contain small enough perturbations of discrete sets of $k$-balls. However, this method does not reveal whether it is possible to prescribe the discrete set of $k$-balls in advance. Initial studies of the ball embedding property were done in 3 , although the notion was not used at that time. Let us state the two theorems of that paper using this notion.

Theorem 2.1 (3, Theorem I). Let $X$ be a complex space with $0<2 \operatorname{dim} X<n$ and suppose that $X$ admits proper holomorphic embedding into $\mathbb{C}^{n}$. Then $X$ satisfies the ball embedding property in $\mathbb{C}^{n}$.

It is a famous theorem of Remmert [9] that any Stein manifold admits proper holomorphic embedding into $\mathbb{C}^{n}$ for $n>2 \operatorname{dim} X$. Hence, for Stein manifolds the embeddability assumption in the theorem above is superfluous. In particular, Theorem 2.1 assures that $\mathbb{C}^{k}$ satisfies the ball embedding property in $\mathbb{C}^{n}$ for $0<2 k<n$. Moreover, it follows that the open unit disc in $\mathbb{C}$ satisfies the ball embedding property in $\mathbb{C}^{n}, n>2$. 
In the dimensional cases not covered by Theorem 2.1 there is a necessary condition for a Stein manifold to satisfy the ball embedding property.

Theorem 2.2 ([3], Theorem II). Let $X$ be a Stein manifold with $0<\operatorname{dim} X<n \leq$ $2 \operatorname{dim} X$. If $X$ satisfies the ball embedding property in $\mathbb{C}^{n}$, then $X$ is $(n-\operatorname{dim} X)$ Eisenman hyperbolic.

This result implies that $\mathbb{C}^{k}$ does not satisfy the ball embedding property in $\mathbb{C}^{n}$ for $0<k<n \leq 2 k$. Indeed, for such $k$ and $n$, the space $\mathbb{C}^{k}$ is not $(n-k)$-Eisenman hyperbolic since $\mathbb{C}^{n-k}$ is trivially included into $\mathbb{C}^{k}(n-k \leq k)$. Thus, Theorems 2.1 and 2.2 give a complete characterisation of the ball embedding property for complex Euclidean spaces: $\mathbb{C}^{k}$ satisfies the ball embedding property in $\mathbb{C}^{n}$ if and only if $0<2 k<n$. In particular, $\mathbb{C}$ does not satisfy the ball embedding property in $\mathbb{C}^{2}$.

The main motivation behind the result in this paper is to demonstrate that the implication in Theorem 2.2 is not trivially true, i.e., we verify the existence of a Stein manifold $X$ and a positive integer $n$ such that $X$ satisfies the ball embedding property in $\mathbb{C}^{n}$ and $0<\operatorname{dim} X<n \leq 2 \operatorname{dim} X$.

\section{Proof of Theorem 1.2}

Let us begin by describing the idea of the proof of Theorem 1.2. Given a discrete set of 1-balls in $\mathbb{C}^{2}$, and $\varepsilon \in \mathbb{R}_{+}^{\infty}$, we begin by considering the image $M$ of a proper holomorphic embedding of $\mathbb{C}$ into $\mathbb{C}^{2}$. By the use of Andersén-Lempert theory we inductively move pieces of $M$ close enough to the 1-balls with the help of holomorphic automorphisms of $\mathbb{C}^{2}$. During this process, we make sure to move a specific point $p \in M$ towards infinity.

The composition of the chosen automorphisms is guaranteed to converge on an open Runge domain $\Omega$ in $\mathbb{C}^{2}$, and the resulting map $\Omega \rightarrow \mathbb{C}^{2}$ is biholomorphic. By construction, the composition does not converge at the point $p \in M$. Thus, the set $\Omega$ is a proper subset of $\mathbb{C}^{2}$ and therefore $\Omega \cap M$ is the union of simply connected open subsets of $M$. We are able to guarantee that the pieces of $\Omega \cap M$ which pass close to the discrete set of 1-balls lie in the same component of $\Omega \cap M$. By the Riemann mapping theorem, this component is biholomorphic to a properly embedded open unit disc. This gives the required proper holomorphic embedding.

We will now carry out the details of the proof. Let $D=\bigcup_{j} D_{j}$ be a discrete set of 1-balls in $\mathbb{C}^{2}$, and let $\varepsilon \in \mathbb{R}_{+}^{\infty}$. Below, we will refer to 1-balls in $\mathbb{C}^{2}$ as discs. Set $M=\mathbb{C} \times\{0\} \subset \mathbb{C}^{2}$ and let $p \in M \backslash\{0\}$ be any point. For each non-negative integer $j$, our aim is to inductively construct

(1) an exhaustion of $\mathbb{C}^{2}$ by connected, compact, and polynomially convex sets $K_{0} \subset K_{1} \subset K_{2} \subset \cdots \subset \bigcup_{j} K_{j}=\mathbb{C}^{2}$ with $K_{j} \subset$ Int $K_{j+1}$,

(2) an increasing sequence of simply connected, compact sets $C_{0} \subset C_{1} \subset C_{2} \subset$ $\cdots \subset \bigcup_{j} C_{j} \subset M \backslash\{p\}$ with $C_{j} \subset \operatorname{Int} C_{j+1}$,

(3) a sequence $\nu_{0}, \nu_{1}, \nu_{2}, \ldots$ of positive numbers, and

(4) a sequence $\varphi_{0}, \varphi_{1}, \varphi_{2}, \ldots$ of holomorphic automorphisms on $\mathbb{C}^{2}$

such that, for $\Phi_{j}:=\varphi_{j} \circ \varphi_{j-1} \circ \cdots \circ \varphi_{0}$, the following properties hold true for all positive integers $l$ :

(a $\left.\mathrm{a}_{j}\right) D_{l} \cap \operatorname{Int} K_{j} \neq \varnothing$ or $\bar{D}_{l} \cap K_{j}=\varnothing$,

( $\left.\mathrm{b}_{j}\right) \Phi_{j-1}\left(C_{j-1}\right) \subset \operatorname{Int} K_{j}$, 
$\left(\mathrm{c}_{j}\right) K_{j} \cap \Phi_{j}(M) \subset \Phi_{j}\left(C_{j}\right)$,

$\left(\mathrm{d}_{j}\right) \bar{D}_{l} \cap \Phi_{j}\left(C_{j}\right)=\varnothing$ for each $D_{l} \subset \mathbb{C}^{2} \backslash K_{j}$,

$\left(\mathrm{e}_{j}\right) \nu_{j}<\min \left\{\varepsilon_{l} / 2 ; l \in \mathbb{N}, D_{l} \subset K_{j}\right\}$,

$\left(\mathrm{f}_{j}\right) \nu_{j} \leq \nu_{j-l} 2^{-l}$ for $l \leq j$,

$\left(\mathrm{g}_{j}\right) \nu_{j}<\operatorname{dist}\left(K_{j-2} \cup \Phi_{j-2}\left(C_{j-2}\right), \mathbb{C}^{2} \backslash\left(K_{j-1} \cup \Phi_{j-1}\left(C_{j-1}\right)\right)\right)$ for $j>1$,

$\left(\mathrm{h}_{j}\right)\left|\varphi_{j}(z)-z\right|<\nu_{j}$ for each $z \in K_{j-1} \cup \Phi_{j-1}\left(C_{j-1}\right), \quad j>0$, and

$\left(\mathrm{i}_{j}\right)$ if $D_{l} \cap K_{j} \neq \varnothing$, then $\Phi_{j}\left(C_{j}\right)$ contains a $\mu_{l}^{j}$-perturbation of $D_{l}$, where $\mu_{l}^{j}:=\sum_{m=j_{l}}^{j} \nu_{m}$ for $j_{l}:=\min \left\{m \in \mathbb{N} ; D_{l} \cap K_{m} \neq \varnothing\right\} \leq j$.

Whenever we need to refer to many of the properties above, which only differ by index, we will omit the index. For example we would simply refer to (f) in order to motivate the statement $\sum_{j=0}^{\infty} \nu_{j}<+\infty$.

Proposition 3.1 ([6], Proposition 5.1). Let $D$ be a connected open set in $\mathbb{C}^{n}$ which is exhausted by compact sets $K_{0} \subset K_{1} \subset K_{2} \subset \cdots \subset \bigcup_{j=0}^{\infty} K_{j}=D$ such that $K_{j-1} \subset \operatorname{Int} K_{j}$ for each $j \in \mathbb{N}$. Choose numbers $\varepsilon_{j}(j=1,2,3, \ldots)$ such that

$$
0<\varepsilon_{j}<\operatorname{dist}\left(K_{j-1}, \mathbb{C}^{n} \backslash K_{j}\right) \quad(j \in \mathbb{N}), \quad \sum_{j=1}^{\infty} \varepsilon_{j}<\infty .
$$

Suppose that for each $j=1,2,3, \ldots, \Psi_{j}$ is a holomorphic automorphism of $\mathbb{C}^{n}$ satisfying

$$
\left|\Psi_{j}(z)-z\right|<\varepsilon_{j}, \quad z \in K_{j}
$$

Set $\Phi_{m}=\Psi_{m} \circ \Psi_{m-1} \circ \cdots \circ \Psi_{1}$. Then there is an open set $\Omega \subset \mathbb{C}^{n}$ such that $\lim _{m \rightarrow \infty} \Phi_{m}=\Phi$ exists on $\Omega$ (uniformly on compacts) and $\Phi$ is a biholomorphic map of $\Omega$ onto $D$. In fact, $\Omega=\bigcup_{m=1}^{\infty} \Phi_{m}^{-1}\left(K_{m}\right)$.

Let us begin by showing that it is enough to construct the desired objects. Set $\Phi:=\lim \Phi_{j}$. According to Proposition 3.1 (with $\varepsilon_{j}, \Psi_{j}$, and $K_{j}$ replaced by $\nu_{j+1}, \varphi_{j+1}$, and $K_{j} \cup \Phi_{j}\left(C_{j}\right)$ resp.), it follows from (f), (g), and (h) that $\Phi: \Omega \rightarrow \mathbb{C}^{2}$ is well-defined and biholomorphic, where

$$
\Omega=\bigcup_{j} \Phi_{j}^{-1}\left(K_{j}\right) \cup C_{j} .
$$

By construction, the map $\Phi: \Omega \rightarrow \mathbb{C}^{2}$ can be approximated by automorphisms of $\mathbb{C}^{2}$, and therefore $\Omega$ is Runge in $\mathbb{C}^{2}$ (see e.g. 8], Proposition 1.2). From (c) it follows that $\Phi_{j}(p) \notin K_{j}$ for any $j$ (since $p \notin C_{j}$ ), i.e., $p \notin \Omega$. Hence, $\Omega \cap M$ is a proper subset of $M$, and it consists of open Runge domains in $M$. Therefore, each such domain is simply connected in $M$. Since $\Phi$ converges on the connected set $C:=\bigcup_{j} C_{j}$, the set $C$ is contained in a single connected component $\Omega^{\prime}$ of $\Omega \cap M$. By applying the Riemann mapping theorem we find a biholomorphic map $G$ from the open unit disc in $\mathbb{C}$ onto $\Omega^{\prime}$. Thus, $F:=\Phi \circ G$ is a proper holomorphic embedding of $\triangle$ into $\mathbb{C}^{2}$. Finally, consider a disc $D_{l}$. For large enough $j$, it follows from $\left(\mathrm{i}_{j}\right)$ that $\Phi_{j}\left(\Omega^{\prime}\right)$ contains a $\mu_{l}^{j}$-perturbation of $D_{l}$. By use of (e) and (f) we see that $\mu_{l}^{j} \leq 2 \nu_{j_{l}}<\varepsilon_{l}$ ( $j$ large enough). Therefore, (i) guarantees that the image $F(\triangle)=\Phi \circ G(\triangle)$ contains an $\varepsilon_{l}$-perturbation of $D_{l}$ for each $l$.

Let us now construct the desired objects. We may assume that the origin is not in the closure of any of the discs $D_{j}$. Let $B \subset \mathbb{C}^{n}$ denote the open unit ball centred at the origin. Choose real numbers $0<r_{0}<r_{0}^{\prime}$ small enough such that $p \notin r_{0}^{\prime} \bar{B}$ 
and $\bar{D}_{j} \cap r_{0}^{\prime} \bar{B}=\varnothing$ for all positive integers $j$. Set $K_{0}:=r_{0} \bar{B}, C_{0}:=M \cap r_{0}^{\prime} \bar{B}$, $\nu_{0}:=1$, and let $\varphi_{0}$ be the identity map on $\mathbb{C}^{2}$.

Let $k$ be a non-negative integer and assume that for any integer $0 \leq j \leq k$ we have constructed $K_{j}, C_{j}, \nu_{j}$, and $\varphi_{j}$ according to (1)-(4) with the properties required in $\left(\mathrm{a}_{j}\right)-\left(\mathrm{i}_{j}\right)$. Moreover, assume that the compact sets $K_{j}$ are given by $K_{j}=$ $r_{j} \bar{B}$, where $r_{j}$ are real numbers so that $r_{j}>r_{j-1}(j>0)$ and $r_{1}>r_{0}^{\prime}>r_{0}$ (according to the above). Set $M_{j}:=\Phi_{j}(M)$ and $p_{j}:=\Phi_{j}(p)$. We will now construct $K_{k+1}, \nu_{k+1}, \varphi_{k+1}$, and $C_{k+1}$.

Let $r_{k+1}>r_{k}$ be large enough so that $\Phi_{k}\left(C_{k}\right) \subset r_{k+1} B$,

$$
D_{l} \cap r_{k+1} B \neq \varnothing \quad \text { or } \quad \bar{D}_{l} \cap r_{k+1} \bar{B}=\varnothing \quad(l=1,2,3, \ldots)
$$

and

$$
D_{l} \cap K_{k}=\varnothing \quad \text { or } \quad \bar{D}_{l} \subset r_{k+1} B \quad(l=1,2,3, \ldots) .
$$

Then the set $K_{k+1}:=r_{k+1} \bar{B}$ is as in (1), and $\left(\mathrm{a}_{k+1}\right),\left(\mathrm{b}_{k+1}\right)$ are satisfied. Now that we have defined $K_{k+1}$, we introduce the index sets

$$
L:=\left\{l \in \mathbb{N} ; D_{l} \cap K_{k} \neq \varnothing\right\}, \quad \tilde{L}:=\left\{l \in \mathbb{N} \backslash L ; D_{l} \cap K_{k+1} \neq \varnothing\right\} .
$$

Observe that the indices in $L$ correspond to discs $D_{l}$ from previous steps of the induction. Indeed, by the induction assumption we are guaranteed that $C_{k} \subset M$ is such that $\Phi_{k}\left(C_{k}\right)$ contain a small enough perturbation of $D_{l}, l \in L$. The indices in $\tilde{L}$ correspond to the new discs which are considered in the current step of the induction.

Before we choose $\nu_{k+1}$, we define sets $E_{k+1}$ and $U_{k+1}$ upon which the choice of $\nu_{k+1}$ will depend. These two sets will also be important for the choice of $C_{k+1}$ below. Set

$$
E_{k+1}:=\bigcup_{l \in \tilde{L}} \bar{D}_{l} \cup K_{k+1} .
$$

Each disc $D_{l}$ defines an affine complex line $P_{l}$ in $\mathbb{C}^{2}$. Observe that $P:=\bigcup_{l \in \tilde{L}} P_{l}$ is a complex subspace of $\mathbb{C}^{2}$ and $A:=E_{k+1} \cap P$ is holomorphically convex in $P$.

Lemma 3.2 ([13], Lemma 2). Let $K \subset \mathbb{C}^{k}$ be polynomially convex, let $V \subset \mathbb{C}^{k}$ be a closed subvariety, and let $K^{\prime} \subset V$ be compact such that $K \cap V \subset K^{\prime}$. Then we have

$$
\widehat{K \cup K^{\prime}}=K \cup \hat{K}_{\mathcal{O}(V)}^{\prime}=K \cup \hat{K}^{\prime}
$$

Since $K_{k+1} \cap P \subset A$, it follows from Lemma 3.2 that $E_{k+1}=K_{k+1} \cup A$ is polynomially convex in $\mathbb{C}^{2}$. Let $U_{k+1}$ be any small enough open neighbourhood of $E_{k+1}$ such that $\bar{U}_{k+1}$ is a compact, polynomially convex set and

$$
\bar{D}_{l} \cap \bar{U}_{k+1}=\varnothing, \quad l \notin L \cup \tilde{L} .
$$

Let us now choose $\nu_{k+1}$. The conditions $\left(\mathrm{e}_{k+1}\right)-\left(\mathrm{g}_{k+1}\right)$ give a finite number of upper bounds for the choice of $\nu_{k+1}$. Thus, we simply choose $\nu_{k+1}$ small enough to meet these requirements. Additionally, we make sure that $\nu_{k+1}$ is small enough so that

$$
\left\{z \in \mathbb{C}^{n} ; \operatorname{dist}\left(z, E_{k+1}\right) \leq \nu_{k+1}\right\} \subset U_{k+1} .
$$

Consider $K_{k} \cup \Phi_{k}\left(C_{k}\right)$. Since $\Phi_{k}\left(C_{k}\right)$ is a compact, holomorphically convex subset of $M_{k}$, and $K_{k} \cap M_{k} \subset \Phi_{k}\left(C_{k}\right)$ according to $\left(\mathrm{c}_{k}\right)$, it follows from Lemma 3.2 that $K_{k} \cup \Phi_{k}\left(C_{k}\right)$ is polynomially convex in $\mathbb{C}^{2}$. This is crucial for the application 
of the Andersén-Lempert theory which is at the heart of constructing a suitable automorphism $\varphi_{k+1}$. We choose $\varphi_{k+1}$ according to the following lemma.

Lemma 3.3. Using the notation above, there exists a holomorphic automorphism $\varphi_{k+1}$ of $\mathbb{C}^{2}$ such that

(i) $\varphi_{k+1}\left(M_{k}\right)$ contains a $\left(\mu_{l}^{k}+\nu_{k+1}\right)$-perturbation of $D_{l}$ for each $l \in L$,

(ii) $\varphi_{k+1}\left(M_{k}\right)$ contains a $\nu_{k+1}$-perturbation of $D_{l}$ for each $l \in \tilde{L}$,

(iii) $\varphi_{k+1}\left(p_{k}\right) \notin \bar{U}_{k+1}$, and

(iv) $\left|\varphi_{k+1}(z)-z\right|<\nu_{k+1}$ for all $z \in K_{k} \cup \Phi_{k}\left(C_{k}\right)$.

Remark 3.4. Lemma 3.3 essentially corresponds to [3], Lemma 3.3, in the special case $k=1$ and $n=2$. In turn, these lemmata are modified versions of [6], Lemma 6.8.

Proof of Lemma 3.3. We will construct two maps $\alpha, \beta \in \operatorname{Aut}\left(\mathbb{C}^{2}\right)$ so that their composition $\varphi_{k+1}:=\beta \circ \alpha$ has the required properties. Recall that $K_{k} \cup \Phi_{k}\left(C_{k}\right)$ is a compact, polynomially convex set in $\mathbb{C}^{2}$. Set $D_{\tilde{L}}:=\bigcup_{l \in \tilde{L}} \bar{D}_{l}$. The set $K:=$ $K_{k} \cup \Phi_{k}\left(C_{k}\right) \cup D_{\tilde{L}}$ is polynomially convex. This follows e.g. by repeated use of Lemma 3.2, adding one disc at a time.

Recall the definition of $U_{k+1}$ (introduced in the proof of the main theorem). Fix a point $x \in \mathbb{C}^{2} \backslash \bar{U}_{k+1}$. Since $K$ is polynomially convex, so is $K \cup\{x\}$. Moreover, this means that $K \cup\{x\}$ has a Stein neighbourhood basis. Thus we may choose an arbitrarily small Runge neighbourhood of $K \cup\{x\}$ with the same number of components as $K \cup\{x\}$. Let $V$ be such a neighbourhood, denoted $V=\bigcup_{l \in \tilde{L}} V_{l} \cup$ $V_{0} \cup V_{x}$, where $V_{0} \supset K_{k} \cup \Phi_{k}\left(C_{k}\right), V_{x} \ni x$, and $V_{l} \supset \bar{D}_{l}(l \in \tilde{L})$ have pairwise disjoint closures. Additionally, we may assume that $V \backslash V_{x} \subset U_{k+1}$.

Next, we explain how to construct the holomorphic automorphism $\alpha \in \operatorname{Aut}\left(\mathbb{C}^{2}\right)$. Let $q_{l} \in \mathbb{C}^{2}$ denote the centre of the disc $D_{l}$. For $l \in \tilde{L}$, choose distinct points $a_{l} \in M_{k} \backslash\left(\left\{p_{k}\right\} \cup \Phi_{k}\left(C_{k}\right)\right)$. Fix any open set $W \Subset V_{0}$ containing $K_{k} \cup \Phi_{k}\left(C_{k}\right)$.

Proposition 3.5 ([6], Proposition 2.1). Let $n>1$. Assume that

(a) $K \subset \mathbb{C}^{n}$ is a compact polynomially convex set,

(b) $\left\{a_{j}\right\}_{j=1}^{s} \subset K$ is a finite subset of $K$,

(c) $p$ and $q$ are arbitrary points in $\mathbb{C}^{n} \backslash K$ (not necessarily distinct),

(d) $N$ is a non-negative integer, and

(e) $P$ : $\mathbb{C}^{n} \rightarrow \mathbb{C}^{n}$ is a holomorphic polynomial map of degree at most $m \geq 1$ with $P(0)=0$ and $J P(0) \neq 0$.

Then for each $\varepsilon>0$ there exists an automorphism $F \in \operatorname{Aut}\left(\mathbb{C}^{n}\right)$ satisfying

(i) $F(p)=q$ and $F(z)=q+P(z-p)+O\left(|z-p|^{m+1}\right)$ as $z \rightarrow p$,

(ii) $F(z)=z+O\left(\left|z-a_{j}\right|^{N}\right)$ as $z \rightarrow a_{j}$ for each $j=1,2, \ldots, s$, and

(iii) $|F(z)-z|+\left|F^{-1}(z)-z\right|<\varepsilon$ for each $z \in K$.

If, in addition, the polynomial map $P$ satisfies

( $\left.\mathrm{e}^{\prime}\right) J P(z)=1+O\left(|z|^{m}\right)$ as $z \rightarrow 0$,

we may choose $F$ to be a polynomial automorphism with Jacobian one.

Through repeated use of Proposition 3.5 (once for each of the points $a_{l}$ and $x$ ), we find a map $\alpha \in \operatorname{Aut}\left(\mathbb{C}^{2}\right)$ such that

$\left(1_{\alpha}\right) \alpha\left(p_{k}\right)=x$,

$\left(2_{\alpha}\right) \alpha\left(a_{l}\right)=q_{l}, \quad l \in \tilde{L}$, 
$\left(3_{\alpha}\right) T_{q_{l}} \alpha\left(M_{k}\right)=T_{q_{l}} P_{l}, \quad l \in \tilde{L}$,

$\left(4_{\alpha}\right) \alpha\left(K_{k} \cup \Phi_{k}\left(C_{k}\right)\right) \subset W$,

$\left(5_{\alpha}\right)|\alpha(z)-z|<\nu_{k+1} / 2, \quad z \in K_{k} \cup \Phi_{k}\left(C_{k}\right)$, and

$\left(6_{\alpha}\right) \alpha\left(M_{k}\right)$ contains a $\left(\mu_{l}^{k}+\nu_{k+1} / 2\right)$-perturbation of $D_{l}, \quad l \in L$.

The use of Proposition 3.5 assures that the modulus in $\left(5_{\alpha}\right)$ can be made arbitrarily small on $K_{k} \cup \Phi_{k}\left(C_{k}\right)$. Thus, we may require that $\alpha$ is arbitrarily close to the identity in $\mathcal{C}^{1}$ norm. Let us verify $\left(6_{\alpha}\right)$. In view of $\left(\mathrm{i}_{k}\right)$, we know that $\Phi_{k}\left(C_{k}\right)$ contains a $\mu_{l}^{k}$-perturbation of $D_{l}$ for each $l \in L$. This means that $\Phi_{k}\left(C_{k}\right)$ contains some graph of a holomorphic function on $D_{l}$. Thus, for $\|\alpha-\mathrm{id}\|_{\mathcal{C}^{1}}$ small enough, $\alpha \circ \Phi_{k}\left(C_{k}\right)$ must contain some graph of a holomorphic function on $D_{l}$. Moreover, this graph cannot differ much from the one contained in $\Phi_{k}\left(C_{k}\right)$. Recall that $\Phi_{k}\left(C_{k}\right) \subset M_{k}$.

Let us now show how to construct an appropriate $\beta \in \operatorname{Aut}\left(\mathbb{C}^{2}\right)$. It will be done with the help of the following result.

Lemma 3.6 ([8, Lemma 1.4). Let $X$ be a holomorphic vector field (resp. divergence zero vector field) defined on all of $\mathbb{C}^{n}$. Let $\Omega$ be an open subset of $\mathbb{C}^{n}$ and let $t_{0}>0$. Assume that the differential equation $d R / d t=X(R(t))$ can be integrated for $0 \leq t \leq t_{0}$ with arbitrary initial condition $R(0)=z \in \Omega$. Set $F_{t}(z)=R(t)$ as above. Then $F_{t}\left(0 \leq t \leq t_{0}\right)$ is a biholomorphic map from $\Omega$ into $\mathbb{C}^{n}$ that can be approximated, uniformly on compact sets in $\Omega$, by automorphisms of $\mathbb{C}^{n}$ (resp. by automorphisms with Jacobian one).

We will define a holomorphic vector field $X$ on the Runge set $V$. This vector field can be approximated, uniformly on a compact subset of $V$, by a global vector field, and then we apply Lemma 3.6 to find a holomorphic automorphism of $\mathbb{C}^{2}$.

Let the restriction of $X$ to $V_{0} \cup V_{x}$ be the zero vector field. Fix an index $l \in$ $\tilde{L}$. After a suitable change of coordinates, we may assume that $q_{l}$ is the origin of $\mathbb{C}^{2}$ and $P_{l}=\mathbb{C} \times\{0\}$. Let $s_{l}>0$ and define the restriction of $X$ to $V_{l}$ by $X \mid V_{l}(z, w):=\left(s_{l} z,-s_{l} w\right)$. The time-one flow of this vector field is given by $F_{X}(z, w)=\left(e^{s_{l}} z, e^{-s_{l}} w\right)$. By choosing $s_{l}$ large enough, it follows from $\left(3_{\alpha}\right)$ that the time-one flow $F_{X}$ stretches a small neighbourhood of $q_{l}$ in $\alpha\left(M_{k}\right)$ onto a small enough perturbation of $D_{l}$. We choose $s_{l}$ large enough to guarantee that a part of $\alpha\left(M_{k}\right)$ is stretched onto a small enough perturbation of $D_{l}$.

In view of Lemma 3.6. the time-one flow of $X$ can be approximated, uniformly on $\bar{W} \cup\{x\}$, by an automorphism of $\mathbb{C}^{2}$. In this way we find $\beta \in \operatorname{Aut}\left(\mathbb{C}^{2}\right)$ such that

$\left(1_{\beta}\right) \beta(x) \in V_{x}$,

$\left(2_{\beta}\right)\|\beta-\mathrm{id}\|_{\mathcal{C}^{1}}$ is small enough on $\bar{W}$, and

$\left(3_{\beta}\right) \beta \circ \alpha\left(M_{k}\right)$ contains $\left(\nu_{k+1}\right)$-perturbations of $D_{l}, l \in \tilde{L}$.

It remains to verify that $\varphi_{k+1}:=\beta \circ \alpha$ has the required properties. By arguments similar to those used to verify $\left(6_{\alpha}\right)$, Lemma 3.3 (i) follows from $\left(4_{\alpha}\right),\left(6_{\alpha}\right)$, and $\left(2_{\beta}\right)$. Lemma 3.3 (ii) is guaranteed by $\left(3_{\beta}\right)$. Since $V_{x} \cap \bar{U}_{k+1}=\varnothing$, the statement in Lemma [3.3 (iii) follows from $\left(1_{\alpha}\right)$ and $\left(1_{\beta}\right)$. Lemma 3.3(iv) follows immediately from $\left(4_{\alpha}\right),\left(5_{\alpha}\right)$, and $\left(2_{\beta}\right)$.

Lemma 3.3(iv) assures that $\varphi_{k+1}$ satisfies $\left(\mathrm{h}_{k+1}\right)$, and it remains to define the set $C_{k+1}$ in such a way as to assure $\left(\mathrm{c}_{k+1}\right),\left(\mathrm{d}_{k+1}\right)$, and $\left(\mathrm{i}_{k+1}\right)$. The key to defining $C_{k+1}$ is to use $U_{k+1}$. Set

$$
F_{k+1}:=\Phi_{k+1}^{-1}\left(\bar{U}_{k+1}\right) \cap M,
$$


and recall that $\bar{U}_{k+1}$ is polynomially convex in $\mathbb{C}^{2}$. Thus, $F_{k+1}$ is holomorphically convex in $M$. Since $M \simeq \mathbb{C}$, this means that $F_{k+1}$ consists of finitely many simply connected components.

Our aim is to choose $C_{k+1}$ so that $F_{k+1} \subset \operatorname{Int} C_{k+1}$. Moreover, we must make sure that $C_{k+1}$ avoids the point $p$ as well as $\Phi_{k+1}^{-1}\left(D_{l}\right), l \notin L \cup \tilde{L}$. Set

$$
\tilde{D}:=\bigcup_{l \notin L \cup \tilde{L}} \bar{D}_{l} .
$$

For each $l \notin L \cup \tilde{L}$, the set $\Phi_{k+1}^{-1}\left(D_{l}\right) \cap M$ is either a finite set of points or $\Phi_{k+1}^{-1}\left(D_{l}\right) \subset$ $M$ is an open, simply connected subset. Since $D$ is a discrete set of discs, it follows from $(\dagger)$ that $F_{k+1}$ cannot contain accumulation points of the set $\Phi_{k+1}^{-1}(\tilde{D}) \cap M$. Moreover, by Lemma 3.3(iii) we have $p \notin F_{k+1}$. Let $C_{k+1}$ be the closure of any small enough, simply connected, open neighbourhood of $F_{k+1}$ in $M \backslash\{p\}$. Then $C_{k+1}$ does not intersect or contain accumulation points of $\Phi_{k+1}^{-1}(\tilde{D}) \cap M$. This proves that $\left(\mathrm{d}_{k+1}\right)$ is satisfied.

Next, we explore the important inclusion $F_{k+1} \subset \operatorname{Int} C_{k+1}$. It implies $\left(\mathrm{c}_{k+1}\right)$, since $K_{k+1} \subset U_{k+1}$. Moreover, we have $\Phi_{k}\left(C_{k}\right) \subset K_{k+1} \subset E_{k+1}$ by use of $\left(\mathrm{b}_{k}\right)$. According to $\left(\mathrm{h}_{k+1}\right)$, it follows from $(\ddagger)$ that $\Phi_{k+1}\left(C_{k}\right)=\varphi_{k+1}\left(\Phi_{k}\left(C_{k}\right)\right) \subset U_{k+1}$. This gives $C_{k} \subset F_{k+1}$. In combination with $F_{k+1} \subset \operatorname{Int} C_{k+1}$, we conclude that $C_{k} \subset \operatorname{Int} C_{k+1}$.

Now it remains to prove that $C_{k+1}$ satisfies $\left(\mathrm{i}_{k+1}\right)$, and we will consider the disjoint cases $l \in L$ and $l \in \tilde{L}$. Let us begin with $l \in \tilde{L}$. According to Lemma 3.3(ii), the image $\Phi_{k+1}(M)=\varphi_{k+1}\left(M_{k}\right)$ contains a $\nu_{k+1}$-perturbation $D_{l}^{\prime}$ of $D_{l}$. It follows from $(\ddagger)$ that $D_{l}^{\prime} \subset U_{k+1}$, and thus $D_{l}^{\prime} \subset \Phi_{k+1}\left(C_{k+1}\right)$. Observe that $\mu_{l}^{k+1}=\nu_{k+1}$ for $l \in \tilde{L}$. Thus, $\left(\mathrm{i}_{k+1}\right)$ is verified for $l \in \tilde{L}$. Next, suppose that $l \in L$. It follows from $\left(\mathrm{i}_{k}\right)$ that $\Phi_{k}\left(C_{k}\right)$ contains a $\mu_{l}^{k}$-perturbation of $D_{l}$. By Lemma 3.3)(i), $\Phi_{k+1}\left(C_{k}\right)$ contains a $\left(\mu_{l}^{k}+\nu_{k+1}\right)$-perturbation of $D_{l}$. According to the definition of $\mu_{l}^{j}$, we have $\mu_{l}^{k+1}=\mu_{l}^{k}+\nu_{k+1}$. Since $C_{k} \subset C_{k+1}$, we conclude that $\Phi_{k+1}\left(C_{k+1}\right)$ contains a $\mu_{l}^{k+1}$-perturbation of $D_{l}$. This proves $\left(\mathrm{i}_{k+1}\right)$ for $l \in L$ and shows that $C_{k+1}$ satisfies $\left(\mathrm{i}_{k+1}\right)$. The proof is finished.

\section{REFERENCES}

[1] S. S. Abhyankar and T. T. Moh, Embeddings of the line in the plane, J. Reine Angew. Math. 276 (1975), 148-166. MR0379502 (52:407)

[2] S. Borell and F. Kutzschebauch, Non-equivalent embeddings into complex Euclidean spaces, Internat. J. Math. 17 (2006), no. 9, 1033-1046. MR.2274009 (2007i:32028)

[3] S. Borell and F. Kutzschebauch, Embeddings through discrete sets of balls, Ark. Mat. 46 (2008), no. 2, 251-269. MR2430726 (2009f:32016)

[4] G. Buzzard and J. E. Fornæss, An embedding of $\mathbb{C}$ into $\mathbb{C}^{2}$ with hyperbolic complement, Math. Ann. 306 (1996), no. 3, 539-546. MR.1415077 (97k:32038)

[5] D. A. Eisenman, Intrinsic measures on complex manifolds and holomorphic mappings, Memoirs of the American Mathematical Society, no. 96, American Mathematical Society, Providence, R.I. (1970). MR0259165(41:3807)

[6] F. Forstnerič, Interpolation by holomorphic automorphisms and embeddings in $\mathbb{C}^{n}$, J. Geom. Anal. 9 (1999), no. 1, 93-117. MR.1760722 (2001g:32040)

[7] F. Forstnerič, J. Globevnik and J.-P. Rosay, Nonstraightenable complex lines in $\mathbb{C}^{2}$, Ark. Mat. 34 (1996), no. 1, 97-101. MR.1396625 (97b:32033)

[8] F. Forstnerič and J.-P. Rosay, Approximation of biholomorphic mappings by automorphisms of $\mathbb{C}^{n}$, Invent. Math. 112 (1993), no. 2, 323-349. MR1213106 (94f:32032)

[9] R. Remmert, Sur les espaces analytiques holomorphiquement séparables et holomorphiquement convex, C. R. Acad. Sci. Paris 243 (1956), 118-121. MR0079808(18:149c) 
[10] J.-P. Rosay and W. Rudin, Holomorphic maps from $\mathbb{C}^{n}$ to $\mathbb{C}^{n}$, Trans. Amer. Math. Soc. 310 (1988), no. 1, 47-86. MR929658 (89d:32058)

[11] J.-P. Rosay and W. Rudin, Holomorphic embeddings of $\mathbb{C}$ in $\mathbb{C}^{n}$, in Several complex variables (Stockholm, 1987/1988), Math. Notes, 38, Princeton Univ. Press (1993), 563-569. MR.1207881 (94c:32017)

[12] M. Suzuki, Propriétés topologiques des polynômes de deux variables complexes, et automorphismes algébriques de l'espace $\mathbb{C}^{2}$, J. Math. Soc. Japan 26 (1974), 241-257. MR0338423 (49:3188)

[13] E. Fornæss Wold, Fatou-Bieberbach domains, Internat. J. Math. 16 (2005), no. 10, 11191130. MR2182211 (2006e:32020)

Department of Mathematics, University of Berne, Sidlerstrasse 5, CH-3012 Berne, SWITZERLAND

E-mail address: stefan.borell@math.unibe.ch

Current address: Department of Natural Sciences, Engineering and Mathematics, Mid Sweden University, SE-851 70 Sundsvall, Sweden

E-mail address: stefan.borell@miun.se 